\title{
CHEMO SELECTIVE, ROOM TEMPERATURE AND SOLVENT FREE SYNTHESIS OF THIO-ESTERS USING EFFECTIVE SYNERGISTIC CATALYTIC SYSTEM
}

\author{
Hari R. Pawar ${ }^{1}$, Narendra R. Kamble ${ }^{1, *}$ and Vinod T. Kamble ${ }^{2}$ \\ ${ }^{1}$ Department of Chemistry, MES's Abasaheb Garware College, Pune-411004 \\ ${ }^{1}$ Department of Chemistry, PDEA's Waghire College, Saswad, Pune.Maharashtra, India \\ ${ }^{2}$ Organic Chemistry Research Laboratory, Department of Chemistry, Institute of Sciences, \\ Nagpur, Maharashtra, India \\ *E-mail: narendra1499@gmail.com
}

\begin{abstract}
A simple and highly efficient room temperature catalytic protocol was developed towards the synthesis of thioesters from thiols and acyl chlorides under solvent-free conditions. Ambient reaction conditions, shorter reaction time, excellent product yields are notable features of this methodology.

Keywords: Niobium Pentachloride, Silver Salt, Thiols, Acyl Chlorides.
\end{abstract}

(C) RASĀYAN. All rights reserved

\section{INTRODUCTION}

The prime intention behind the development of new methodologies in synthetic organic chemistry has been the need for simple, clean and efficient strategies to obtain diverse target molecules and its analogs. Thio-esters are one of the activated carboxylic acid derivatives which act as potent acylating reagent comparable to those of acid anhydrides ${ }^{1}$ and are vital functionality in organic transformations. ${ }^{2}$ Biologically active thioesters play essential role in as indispensable metabolic intermediates in living cells owing to its ability to work as excellent acyl group transfer in biochemical reactions. In addition, natural synthesis of polypeptides and non-ribosomal polypeptides is achieved by thioester intermediates of amino and fatty acids. ${ }^{3}$

Usually thioesters are prepared by condensation of a thiols and acid chloride and they have diverse applications in synthetic chemistry as precursors to aldehydes, ketones, acids, esters, lactones, amides, lactams and heterocycles. ${ }^{4}$ Surprisingly, in light of diverse chemistry of thioester functionality, pave the way for combinatorial chemists to design organic molecules. ${ }^{5}$ Due to the significance of thioesters, a variety of chemical and enzyme-based methods have been developed for its synthesis from carboxylic acids $^{6}$ or acid chlorides. ${ }^{7}$ The transformation of alkyl halides to thioesters using potassium thioacetate is reported in the literature. ${ }^{8}$ Recently radical initiator based azobis [2-(2-imidazoline-zolyl)] propane dihydrochloride methods are also been developed. Cetyl Trimethyl Ammonium Bromide (CTAB) as surfactant was found to be the most suitable medium for the efficient and direct synthesis thioesters the resultant aldehydes in water. ${ }^{9}$ Thio-esters are employed in $\mathrm{C}-\mathrm{C}$ bond forming reactions and in other functional group transformations. Moreover, they demonstrate higher reactivity and selectivity towards nucleophilic substitution as compared to oxy-esters which makes them the unique acylating agent in chemical synthesis. Classically, thioesters were made from the reaction of carboxylic acids and thiols. ${ }^{10}$ The major drawback of this method is that it requires the activation of acid. Varieties of activating reagents are involved for this purpose such as triasalkylthioborane, ${ }^{11}$ phenyl dichloro phosphonate, ${ }^{12}$ tri-nbutyl phosphine, ${ }^{13}$ diethyl phosphoro cynidate ${ }^{14}$ and triphenylphosphine NBS $/$ NIS $^{15}$ and phosgene ${ }^{16}$ have been reported. Preparation of thioesters from the reaction of amides and thiols using aluminium thiophenoxide or boron thiophenoxide were also been reported in the literature. ${ }^{17}$ However, some other

Rasayan J. Chem., 12(4), 1997-2004(2019)

http://dx.doi.org/10.31788/RJC.2019.1245381

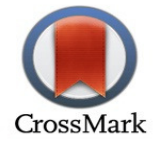


methods involves use of $\mathrm{N}$-methyl benzene thiazolium trifluoro methanesulfonate, ${ }^{18}$ zinc $^{19}$ and thallium ${ }^{20}$ tin $^{21}$ copper-mercaptides ${ }^{22}$ proceeds through acid chloride. ${ }^{23}$ In recent years, cyanuric chloride ${ }^{24}$ and Dess-Martin periodinane ${ }^{25-28}$ have been investigated to catalyze this reaction. All these methodologies have one or the other lacunas such as use of expensive reagents, tedious work-up, hazardous reagents and sometimes which generates secondary wastes.

Strong Lewis acid niobium pentachloride as a useful reagent in organic synthesis because of its stability, less hygroscopic nature and eco-friendly in handling compared to other Lewis acids. In various organic transformations are catalyzed by $\mathrm{NbCl}_{5}$ in acyl bond and C-P bond formation reactions. We have adopted highly chemoselective method of dealkylation of alkyl aryl ethers with a stoichiometric amount of $\mathrm{NbCl}_{5}{ }^{29}$ We have utilized the potentiality of $\mathrm{NbCl}_{5}$ as a catalyst for acylation reaction as the most prominent reactions in organic synthesis. ${ }^{30-32}$

\section{EXPERIMENTAL}

It is noteworthy to mention that Lewis acidity is essential for retaining the catalytic activity towards the sustainability of process in a continuous manner. The present investigation involves $\mathrm{NbCl}_{5}$ catalyst found to be stable and catalytically robust which brings thioester conversion at room temperature in shorter time. The beneficial catalytic activity of catalyst may be due to the Lewis acidic sites present on the surface of $\mathrm{NbCl}_{5}$ which bring activation of acid chlorides and thiols in this synthetic transformation. However, the stoichiometric amount of $\mathrm{AgClO}_{4}$ promotes this conversion in better manner.

The solid acid $\mathrm{NbCl}_{5}$ is found to be a better catalyst because of higher specific surface area, enhanced particle transport and generation of distinct reaction sites. Such a synthetic strategy is further modified by dispersing $\mathrm{AgClO}_{4}$ as promoter. There are three distinct advantages: (i) its acidity can be tuned as per the organic transformations (ii) proper dispersion of reactive sites for the effective molecular collisions; (iii) solid support is effective for stronger adsorption thereby increasing the reaction rate; (iv) plausibility of its continuous usage. These methodologies are regarded as a sustainable approach.

Adopting these views, we have rationalized the highly efficient chemo selective, solvent-free synthesis of thioesters from acid chlorides and thiols in the presence of $\mathrm{NbCl}_{5}$ and $\mathrm{AgClO}_{4}$ as a synergistic catalyst at room temperature.

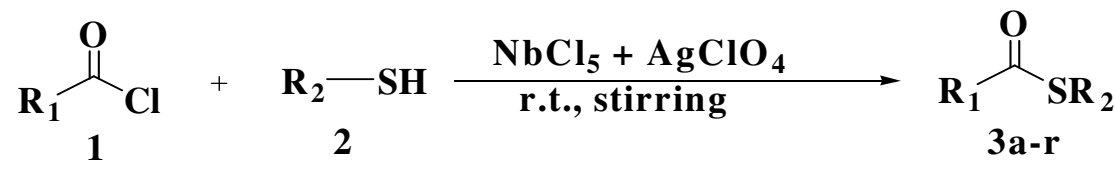

$$
\mathbf{R}_{1} \& \mathbf{R}_{2}=\text { alkyl, aryl }
$$

\section{General Procedure for the Synthesis of Thioester}

Scheme-1

A mixture of acid chloride $(1 \mathrm{mmol}), 1 \mathrm{~mol} \%$ of $\mathrm{NbCl}_{5}, 3 \mathrm{~mol} \%$ of $\mathrm{AgClO}_{4}$, thiol $(1 \mathrm{mmol})$ was prepared and stirred at room temperature. The appearance of yellow-brown color changes to dark with the advancement of the reaction. The reaction mixture was stirred continue up to the completion of the reaction and monitored by TLC. Then it was diluted by adding DCM $10 \mathrm{~mL}$, catalyst was filtered, and washed with $15 \mathrm{~mL}$ DCM. This extract was washed with aqueous sodium bicarbonate and dried over anhydrous sodium sulfate. The solvent was removed under reduced pressure so as to obtain pure products.

\section{Spectral data}

3a:S-phenyl benzothioate

Solid; mp 56-58 ${ }^{\circ}$; IR (KBr): 894, 1200, 1666, 2916, $3059 \mathrm{~cm}^{1} ;{ }^{1} \mathrm{H}$ NMR $\left(\mathrm{CDCl}_{3}, 300 \mathrm{MHz}\right), \delta$; 7.27 7.63(m, $10 \mathrm{H}, \mathrm{Ar}-\mathrm{H}) ;{ }^{13} \mathrm{C} \mathrm{NMR}\left(\mathrm{CDCl}_{3}, 100 \mathrm{MHz}\right), \delta ; 189.9,136.4,135.1,133.4,129.4,129.1,128.5$, 127.4, 127.3; Anal. calcd for $\mathrm{C}_{13} \mathrm{H}_{10} \mathrm{SO}$ : C, 72.86; H, 4.70; S, 14.96; Found: C, 72.85; H, 4.72; S, 14.93 .

\section{3b:S-4-Methylphenyl thiobenzoate}

Solid; mp 64 ${ }^{\circ} \mathrm{C}$; IR (KBr): 645, 897, 1204, 1668, 2916, $3070 \mathrm{~cm}^{-1}$; ${ }^{1} \mathrm{H}$ NMR $\left.\left(\mathrm{CDCl}_{3}\right), 300 \mathrm{MHz}\right), \delta ; 3.38$ (s, 3H, Ar- $\left.\mathrm{CH}_{3}\right), 7.22$ - 7.57 (m, 5H, Ar-H), 8.00 (d, $\left.J=7.2 \mathrm{~Hz}, 2 \mathrm{H}, \mathrm{Ar}-\mathrm{H}\right), 8.10$ (d, $\left.J=7.2 \mathrm{~Hz}, 2 \mathrm{H}, \mathrm{ArH}\right)$; Anal. calcd for $\mathrm{C}_{14} \mathrm{H}_{12} \mathrm{SO}$ : C, 73.65; H, 5.30; S, 14.04; Found: C, 73.61; H, 5.33; S, 14.01. 
RASĀYAN J. Chem.

Vol. 12 | No. 4 |1997 - 2004| October - December | 2019

3c:S-Phenyl 2,2-dimethylthiopropanoate

Solid; mp33-35 C; IR (KBr): 645, 897, 1204, 1668, 2916, $\left.3070 \mathrm{~cm}^{-1} ;{ }^{1} \mathrm{H} \mathrm{NMR} \mathrm{(CDCl} 3,300 \mathrm{MHz}\right), \delta ; 1.58$ $\left(\mathrm{s}, 9 \mathrm{H}, 3 \times \mathrm{CH}_{3}\right), 7.30-7.68(\mathrm{~m}, 5 \mathrm{H}, \mathrm{Ar}-\mathrm{H}) ;{ }^{13} \mathrm{C} \mathrm{NMR}\left(\mathrm{CDCl}_{3}, 100 \mathrm{MHz}\right), \delta ; 204.4,134.9,129.0,128.9$, 128.0, 46.8, 27.3.Anal.calcd for $\mathrm{C}_{11} \mathrm{H}_{14} \mathrm{SO}$ : C, 67.99; H, 7.26; S, 16.50; Found: C, 67.97; H, 7.29; S, 16.47 .

3d:S-4-Methylphenyl 2,2-dimethylthiopropanoate

Solid; mp 380 C; IR (KBr): 620, 805, 929, 1473, 1695, 2930, $2966 \mathrm{~cm}^{-1} ;{ }^{1} \mathrm{H}$ NMR $\left(\mathrm{CDCl}_{3}, 300 \mathrm{MHz}\right), \delta$; $1.60\left(\mathrm{~s}, 9 \mathrm{H}, 3 \times \mathrm{CH}_{3}\right), 2.42$ (s, 3H, Ar- $\left.\mathrm{CH}_{3}\right), 7.99$ (d, $\left.J=7.4 \mathrm{~Hz}, 2 \mathrm{H}, \mathrm{Ar}-\mathrm{H}\right), 8.09$ (d, $\left.J=7.4 \mathrm{~Hz}, 2 \mathrm{H}, \mathrm{Ar}-\mathrm{H}\right)$; Anal. calcd for $\mathrm{C}_{12} \mathrm{H}_{16} \mathrm{SO}$ : C, 69.18; H, 7.74; S, 15.38; Found: C, 69.20; H, 7.71; S, 15.35.

\section{3e:S-Phenyl thioethanoate}

Oil; IR (Neat): 620, 746, 1354, 1439, 1709, 2928, $3062 \mathrm{~cm}^{-1} ;{ }^{1} \mathrm{H}$ NMR $\left(\mathrm{CDCl}_{3}, 300 \mathrm{MHz}\right), \delta ; 2.40$ (s, 3H, $\left.\mathrm{CH}_{3}\right), 7.28$ - $7.76(\mathrm{~m}, 5 \mathrm{H}, \mathrm{Ar}-\mathrm{H}) ;{ }^{13} \mathrm{C} \mathrm{NMR}\left(\mathrm{CDCl}_{3}, 100 \mathrm{MHz}\right) \delta=193.8,134.3,129.3,129.0,127.8$, 30.0; Anal. calcd for $\mathrm{C}_{8} \mathrm{H}_{8} \mathrm{SO}: \mathrm{C}, 63.12 ; \mathrm{H}, 5.30 ; \mathrm{S}, 21.06$; Found: C, 69.17; H, 7.73; S, 15.32 .

\section{3f:S-4-Methylphenyl thioethanoate}

Oil; IR (Neat): 615, 746, 1114, 1478, 1708, 3061 $\mathrm{cm}^{-1} ;{ }^{1} \mathrm{H}$ NMR $\left(\mathrm{CDCl}_{3}, 300 \mathrm{MHz}\right), \delta ; 2.38$ (s, 3H, $\left.\mathrm{COCH}_{3}\right), 3.41\left(\mathrm{~s}, 3 \mathrm{H}, \mathrm{Ar}-\mathrm{CH}_{3}\right), 7.97(\mathrm{~d}, J=7.2 \mathrm{~Hz}, 2 \mathrm{H}, \mathrm{Ar}-\mathrm{H}), 8.01(\mathrm{~d}, J=7.2 \mathrm{~Hz}, 2 \mathrm{H}, \mathrm{Ar}-\mathrm{H})$; Anal. calcd for $\mathrm{C}_{9} \mathrm{H}_{10} \mathrm{SO}$ : C, 65.02; H, 6.06; S, 19.28; Found: C, 65.00; H, 6.02; S, 19.31 .

\section{3g:S-Phenyl thiocinnamate}

Semi-solid;IR (Neat): 670, 895, 1605, 1680, 2900, 3029 $\mathrm{cm}^{-1} ;{ }^{1} \mathrm{H}$ NMR $\left(\mathrm{CDCl}_{3}, 300 \mathrm{MHz}\right), \delta ; 7.21$ (s, 5H, $\mathrm{Ar}-\mathrm{H}), 7.4(\mathrm{~s}, 5 \mathrm{H}, \mathrm{Ar}-\mathrm{H}), 7.5(\mathrm{~d}, 1 \mathrm{H}, J=16.2 \mathrm{~Hz},=\mathrm{CH}), 7.62(\mathrm{~d}, 1 \mathrm{H}, J=16.2 \mathrm{~Hz},=\mathrm{CH})$; Anal. calcd for $\mathrm{C}_{15} \mathrm{H}_{12} \mathrm{SO}$ : C, 74.96; H, 5.03; S, 13.34; Found: C, 74.98; H, 5.06; S, 13.31.

\section{3h:S-4-Methylphenyl thiocinnamate}

Oil;IR (Neat): 690, 880, 1609, 1675, 2917, $3060 \mathrm{~cm}^{-1} ;{ }^{1} \mathrm{H}$ NMR $\left(\mathrm{CDCl}_{3}, 300 \mathrm{MHz}\right), \delta ; 2.3$ (s, 3H, Ar$\left.\mathrm{CH}_{3}\right), 7.1(\mathrm{~s}, 5 \mathrm{H}, \mathrm{Ar}-\mathrm{H}), 7.4(\mathrm{~d}, 1 \mathrm{H}, J=15.5 \mathrm{~Hz},=\mathrm{CH}), 7.55(\mathrm{~d}, 1 \mathrm{H}, J=15.5 \mathrm{~Hz},=\mathrm{CH}), 7.85(\mathrm{~d}, 2 \mathrm{H}, J=$ 7.4Hz, Ar-H), 7.90 (d, $2 \mathrm{H}, J=7.4 \mathrm{~Hz}, \mathrm{Ar}-\mathrm{H})$; Anal. calcd for $\mathrm{C}_{16} \mathrm{H}_{14} \mathrm{SO}: \mathrm{C}, 76.55 ; \mathrm{H}, 5.55 ; \mathrm{S}, 12.60$; Found: C, 76.53; H, 5.51; S, 12.57.

\section{3i: S-Phenyl 4-chlorothiobenzoate}

Solid; $\mathrm{mp} 69^{0} \mathrm{C}$; IR (KBr): 720, 916, 1171, 1588, 1665, 2970, $3035 \mathrm{~cm}^{-1} ;{ }^{1} \mathrm{H} \mathrm{NMR}\left(\mathrm{CDCl}_{3}, 300 \mathrm{MHz}\right), \delta$; 7.03 - $7.52(\mathrm{~m}, 5 \mathrm{H}, \mathrm{Ar}-\mathrm{H}), 7.90(\mathrm{~d}, J=8.5 \mathrm{~Hz}, 2 \mathrm{H}, \mathrm{Ar}-\mathrm{H}), 8.02(\mathrm{~d}, J=8.5 \mathrm{~Hz}, 2 \mathrm{H}, \mathrm{Ar}-\mathrm{H})$; Anal. calcd for $\mathrm{C}_{13} \mathrm{H}_{9} \mathrm{SOCl}$ : C, 62.77; H, 3.65; S, 12.89; Cl, 14.25; Found: C, 62.75; H, 3.63; S, 12.86; Cl, 14.27.

\section{3j:S-4-Methylphenyl 4-chlorothiobenzoate}

Solid; mp 48 ${ }^{0} \mathrm{C}$; IR (KBr): 740, 925, 1170, 1603, 1670, 2960, $3040 \mathrm{~cm}^{-1} ;{ }^{1} \mathrm{H}$ NMR $\left(\mathrm{CDCl}_{3}, 300 \mathrm{MHz}\right), \delta$; $2.30\left(\mathrm{~s}, 3 \mathrm{H}, \mathrm{Ar}-\mathrm{CH}_{3}\right), 7.69(\mathrm{~d}, J=8.5 \mathrm{~Hz}, 2 \mathrm{H}, \mathrm{Ar}-\mathrm{H}), 7.76(\mathrm{~d}, J=8.5 \mathrm{~Hz}, 2 \mathrm{H}, \mathrm{Ar}-\mathrm{H}, \mathrm{Ar}-\mathrm{H}), 7.95(\mathrm{~d}, J=$ 7.4Hz, $2 \mathrm{H}, \mathrm{Ar}-\mathrm{H}), 8.01\left(\mathrm{~d}, J=7.4 \mathrm{~Hz}, 2 \mathrm{H}, \mathrm{Ar}-\mathrm{H}\right.$ ); Anal. calcd for $\mathrm{C}_{14} \mathrm{H}_{11} \mathrm{SOCl}: \mathrm{C}, 63.99 ; \mathrm{H}, 4.22 ; \mathrm{S}$, 12.20; Cl, 13.49; Found: C, 64.02; H, 4.25; S, 12.17; Cl, 13.45 .

\section{3k: S-Phenyl 1,4-dithiobenzoate}

Solid; mp58-60 C; IR (KBr): 751, 845, 1240, 1669, 1690, 2930, 3061 $\mathrm{cm}^{-1} ;{ }^{1} \mathrm{H} \mathrm{NMR}\left(\mathrm{CDCl}_{3}, 300 \mathrm{MHz}\right)$, $\delta ; 7.21$ - $7.54(\mathrm{~s}, 10 \mathrm{H}, \mathrm{Ar}-\mathrm{H}), 7.85(\mathrm{~s}, 4 \mathrm{H}, \mathrm{Ar}-\mathrm{H})$; Anal. calcd for $\mathrm{C}_{20} \mathrm{H}_{14} \mathrm{~S}_{2} \mathrm{O}_{2}$ : C, 68.54; H, 4.29; S, 18.59; Found: C, 68.56; H, 4.31; S, 18.55 .

\section{1: S-Phenyl 4-nitrothiobenzoate}

Solid; mp 98 C; IR (KBr): 742, 850, 1220, 1670, 2918, $3062 \mathrm{~cm}^{-1} ;{ }^{1} \mathrm{H}$ NMR $\left(\mathrm{CDCl}_{3}, 300 \mathrm{MHz}\right), \delta$; 7.31$7.58(\mathrm{~m}, 5 \mathrm{H}, \mathrm{Ar}-\mathrm{H}), 7.92$ (d, $J=7.0 \mathrm{~Hz}, 2 \mathrm{H}, \mathrm{Ar}-\mathrm{H}), 8.03$ (d, $J=7.0 \mathrm{~Hz}, 2 \mathrm{HAr}-\mathrm{H}$,$) ) { }^{13} \mathrm{C} \mathrm{NMR}\left(\mathrm{CDCl}_{3}, 100\right.$ 
MHz) $\delta=188.71,151.6,141.76,135.6,132.10,129.5,128.45,125.11,124.19$; Anal. calcd for $\mathrm{C}_{13} \mathrm{H}_{9} \mathrm{SNO}_{3}$ : C, 60.22; H, 3.50; S, 12.36; N, 5.40; Found: C, 60.25; H, 3.52; S, 12.34; N, 5.41.

\section{RESULTS AND DISCUSSION}

$\mathrm{NbCl}_{5}$ and $\mathrm{AgClO}_{4}$ is synergistic catalyst towards highly effective and chemo selective acylation of thiols in solvent-free environment. The results are presented in (Table-1). This method is recognized to be broad or both aromatic well as aliphatic acid chlorides and brings efficient conversions with aromatic and aliphatic thiols ensuing in the formation of the consequent thioesters with better yields in shorter reaction time. This protocol is remarkably green, clean and involving no tedious work up as well as no chromatographic separation is required to obtain pure products. It is indeed to note that chemo selective acylation occurs absolutely at $\mathrm{S}$ atom and not on the ring carbon atom. The most interesting fact to mention that the reaction happens at room temperature is sufficient enough not promotes cleavage of ether (entry o, Table-1).

\begin{tabular}{|c|c|c|c|c|c|}
\hline Entry & Acid Chloride & Thiol & Product & $\begin{array}{l}\text { Time } \\
(\min .)\end{array}$ & $\begin{array}{c}\text { Yield } \\
(\%)\end{array}$ \\
\hline a & & & & 05 & 94 \\
\hline b & & & & 05 & 96 \\
\hline c & & & & 07 & 96 \\
\hline d & & & & 15 & 90 \\
\hline e & & & & 07 & 95 \\
\hline f & & & & 05 & 92 \\
\hline $\mathbf{g}$ & & & & 35 & 87 \\
\hline h & & & & 32 & 88 \\
\hline $\mathrm{i}$ & & & & 35 & 82 \\
\hline $\mathbf{j}$ & & & & 32 & 83 \\
\hline k & & & & 50 & 85 \\
\hline 1 & & & & 35 & 80 \\
\hline
\end{tabular}


RASĀYAN J. Chem.

Vol. 12 | No. 4 |1997 - 2004| October - December | 2019

\begin{tabular}{|c|c|c|c|c|c|}
\hline Entry & Acid Chloride & Thiol & Product & $\begin{array}{l}\text { Time } \\
(\min .)\end{array}$ & $\begin{array}{l}\begin{array}{l}\text { Yield } \\
(\%)\end{array} \\
\end{array}$ \\
\hline m & & Et-SH & & 15 & 88 \\
\hline n & & Et-SH & & 30 & 90 \\
\hline o & & Et-SH & & 30 & 90 \\
\hline p & & Et-SH & & 45 & 88 \\
\hline$q$ & & Et-SH & & 07 & 75 \\
\hline $\mathbf{r}$ & & & & 15 & 85 \\
\hline
\end{tabular}

Even the dicarboxylic acid chloride too efficiently transformed into the consequent dithio-ester in presence of two equivalents of thiol (entry k, Table-1). The better yields are obtained during the condition when both the reactants are aromatic in nature in shorter reaction times without activation of thiols. Further, we studied the chemo selectivity the acylation by conducting the competitive acylation of phenols, alcohols, amines and thiols at ambient conditions. Interestingly, it was observed that only thiols selectively undergo acylation in presence of phenols, alcohols, and amines (Chart-I). In brief this is simple, efficient protocol towards preparation of thioesters from thiols by employing non-toxic and lowcost $\mathrm{NbCl}_{5}$ as a catalyst without solvent at room temperature condition. The major advantages of this efficient protocol are green which comprises: simple reaction assembly not need of special equipment, less amount of catalyst is adequate to bring the conversion, ambient conditions, better yields of products with excellent chemoselectivity.

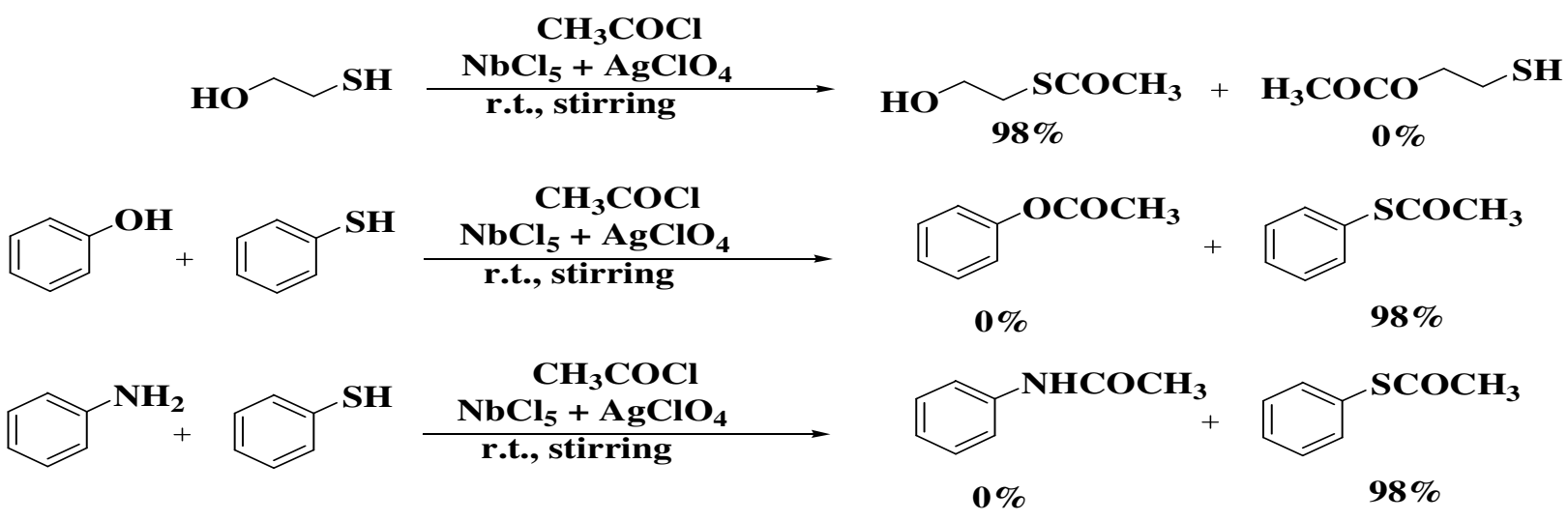

Chart-I

\section{CONCLUSION}

In conclusion, we have investigated a simple, highly efficient and chemo selective method towards acylation of thiols to provide thioesters with use of $\mathrm{NbCl}_{5}$ and $\mathrm{AgClO}_{4}$ as a catalyst at solvent-free condition. This is green and clean method with simple reaction set up offers excellent yields of products with short reaction times at ambient conditions with high selectivity are some of the main advantages of this protocol. 
RASĀYAN J. Chem.

Vol. 12 | No. 4 |1997 - 2004| October - December | 2019

\section{ACKNOWLEDGMENT}

Authors are thankful to Principal, PDEA's Waghire College, Saswad, Pune. Maharashtra, India

\section{REFERENCES}

1. (a) A. Ogava and N. Sonoda, Comprehensive Organic Functional Group Transformations, 5, 231(1995), DOI:10.1016/B0-08-044705-8/00180-1 (b) Peter J. May, Mark Bradley, David C. Harrowven and David Pallin, Tetrahedron Letters, 41(10), 1627(2000), DOI:10.1016/S00404039(99)02345-X (c) D.R. Hogg and G.C. Barrette, Organic Compounds of Sulphur, Selenium and Tellurium, Volume 6, RSC Publications London, 1981, pp. 13-49, DOI: 10.1039/9781847556851-00001

2. (a) D.E. Bublitz, Journal of Organic Chemistry, 32(5), 1630(1967), DOI:10.1021/jo01280a082 (b) G.S. Bates, S. Masamune and J. W. Carcoran, Angewandte Chemie Int. Ed. Engl., 16(9), 585(1977), DOI:10.1002/anie.197705851 (c) T.C. Back, Tetrahedron, 33(23), 3041(1977), DOI: 10.1016/0040-4020(77)80449-3 (d) K. C. Nicolaou, Tetrahedron, 33(7), 683(1977), DOI: 10.1016/0040-4020(77)80180-4 (e) T. Mukaiyama, M. Araki and H. Takei, Journal of American Chemical Society, 95(14), 4763(1973), DOI: 10.1021/ja00795a055 (f) C.J. Chapman, C.G. Frost, J.P. Hartley, A.J. Whittle, Tetrahedron Letters, 42(5), 773(2001), DOI:10.1016/S00404039(00)02122-5 (g) E.J. Corey and S. Bhattachaya, Tetrahedron Letters, 18(45), 3919(1977), DOI: 10.1016/S0040-4039(01)83390-6 (h) H.J. Liu and H.K. Lai, Tetrahedron Letters, 20(14), 1193(1979), DOI: 10.1016/S0040-4039(01)86100-1 (i) M. Jun-ichi, K. Odashima, S. Kobayashi, Synlett, 2000(3), 403(2000), DOI:10.1055/s-2000-6549 (j) Y. Nagao, K. Kawabata, and E. Fujita, Journal of Chemical Society,Chemical Communications 1978(7), 330(1978), DOI: 10.1039/C39780000330 (k) M.L. Wolfform, and J.V. Karabinos, Journal of Americal Chemical Society, 68(4), 742(1946), DOI:10.1021/ja01208a515

3. T.A. Keating and C.T. Walsh, Current Opinion in Chemical Biology, 3(5), 598(1999), DOI: 10.1016/S1367-5931(99)00015-0

4. (a) D.A. Evans, M.J. Dart, J.L. Duffy and M.G. Yang, Journal of American Chemical Society, 118(18), 4322(1996), DOI:10.1021/ja953901u (b) J.D. White, T.S. Kim, and M. Nambu, Journal of American Chemical Society, 119(1), 103 (1997), DOI: 10.1021/ja9629874 (c) M. Mizumo, I. Muramuto, T. Kawakami, M. Soike, S. Aimoto, K. Hanoda and T. Inazu, Tetrahedron Letters, 39(1-2), 55(1998), DOI: 10.1016/S0040-4039(97)10487-7 (d) I.W. Yang, D. Romo, Tetrahedron 53(48), 16471(1997), DOI:10.1016/S0040-4020(97)01029-6 (e) V.K. Agarwal, A. Thomas and S. Schade, Tetrahedron., 53(48), 16213(1997), DOI:10.1016/S0040-4020(97)01010-7 (f) P.J. Um, D.G. Drucckhammer, Journal of American Chemical Society, 120(23), 5605(1998), DOI: 10.1021/ja980445b (g) M.M. Paz, J.F. Corrca, M.I. Cabcza and F.J. Sardina, Tetrahedron Letters, 37(51), 9259(1996), DOI: 10.1016/S0040-4039(96)02138-7

5. (a) L.E. Canne, S.M. Walker, B.H. Stephen, Tetrahedron Letters 36(8), 1217(1996), DOI: 10.1016/0040-4039(95)00037-D (b) S. Kobayashi, I. Iichiya and M. Yasuda, Tetrahedron Letters, 37(31), 5569(1996), DOI:10.1016/0040-4039(96)01158-6 (c) S. Kobayashi, I. Iilachiya, S. Suzuki and M. Moriwaki, Tetrahedron Letters, 37(16), 2809(1996), DOI:10.1016/00404039(96)00435-2

6. (a) T. Cohen and R.E. Gapinski, Tetrahedron Letters, 19(45), 4319(1978), DOI:10.1016/S00404039(01)95213-X (b) A. Pelter, T. Levitt and K. Smith, Journal of Chemical Society D: Chemical Communications, 1969(9), 435(1969), DOI:10.1039/C29690000435 (c) D. Ravi, n. Ram Rao, G.S. Reddy, K. Sucheta, and V.J. Rao, Synlett, 1994(10), 856(1994), DOI: 10.1055/s1994-23031 (d) S. Yamada, Y. Yokuyama and t. Shioiri, Journal of Organic Chemistry, 39(22), 3302(1974), DOI: 10.1021/jo00936a038 (e) P.A. Grieco, Y. Ycoyama, and E. Williams, Journal of Organic Chemistry, 43(6), 1283(1978), DOI:10.1021/jo00400a070 (f) H. Liu and S.I. Sabesan, Canadian Journal of Chemistry, 58(23), 2645(1980), DOI:10.1139/v80-423

7. (a) H.M. Meshram, G.S. Reddy, K.H. Bindu and J.S. Yadav, Synlett, 1998(8), 877(1998), DOI: 10.1055/s-1998-1797 (b) S.T.A. Shaha, K.M. Shan, A.M. Heinrich and W. Voelter, Tetrahedron Letters, 43(46), 8281 (2002), DOI:10.1016/S0040-4039(02)02028-2 (c) A. Padwa, S.J. Coasts 
RASĀYAN J. Chem.

Vol. 12 | No. 4 | 1997 - 2004| October - December | 2019

and L. Hadjarapoglou, Heterocycles, 39(1), 219(1994), DOI: 10.3987/COM-93-S(B)16 (d) H.U. Reibig and B. Scherer, Tetrahedron Letters, 21(44), 4259(1980), DOI:10.1016/S00404039(00)92877-6

8. T. Zheng, M. Burkart and D.E. Richrdson, Tetrahedron Letters, 40(2), 603(1999), DOI: 10.1016/S0040-4039(98)02545-3.

9. H. Nambu, K. Hata, M. Motsugi and Y. Kita, Chemistry a European Journal, 11(2), 719(2005), DOI: $10.1002 /$ chem.200400754

10. A. Pelter, T. Levitt, K. Smith, Journal of Chemical Society D: Chemical Communications, 1969(9), 435(1969), DOI:10.1039/C29690000435

11. H. Jiu and S.I. Sabesan, Canadian Journal of Chemistry, 28(23), 2645(1985), DOI: 10.1139/v80-423

12. P.A. Grieco, Y. Yokoyama, and E. Williams, Journal of Organic Chemistry, 44(13), 2189(1979), DOI: $10.1021 /$ jo01327a033

13. S. Yamada, Y. Yokoyama, and T. Shioiri, Journal of Organic Chemistry, 39(22), 3302(1974), DOI: $10.1021 /$ jo00936a038

14. K. Sucheta, G.S.R. Reddy, D. Ravi, and N. Rama Rao, Tetrahedron Letters, 35(25), 4416(1994), DOI: $10.1016 / \mathrm{S} 0040-4039(00) 73371-5$

15. B. P. Bandger , S. S. Pandit, Journal of Sulfur Chemistry, 25(5), 343(2005), DOI: 10.1080/17415990412331317928

16. D.N. Harpp, T. Aida and T.H. Ghan, Tetrahedron Letters, 20(31), 2853(1979), DOI: 10.1016/S0040-4039(01)86433-9

17. F.S. Bachiller, G.S. Bates, S. Musamune, Journal of Chemical Society, Chemical Communications, 1976(18), 719(1976), DOI: 10.1039/C39760000719.

18. H.M. Meshram, S.G. Reddy, H. Bindu, J.S. Yadav, Synlett, 1998(8), 877(1998), DOI: 10.1055/s-1998-1797

19. S. Musamune, S. Kamata, W. Schilling, Journal of American Chemical Society, 97(12), 3515(1975), DOI: $10.1021 / \mathrm{ja} 00845 \mathrm{a} 039$

20. M.R. Detty, G. P. Wood, Journal of Organic Chemistry, 45(1), 80(1980), DOI: 10.1021/jo01289a018

21. D.N. Harp, T. Aida, T.H. Cham, Tetrahedron Letters, 20(31), 2853(1979), DOI:10.1016/S00404039(01)86433-9

22. H.U. Reibig, B. Scherer, Tetrahedron Letters, 21(44), 4259(1980), DOI:10.1016/S00404039(00)92877-6

23. A. Padwa, S.J. Coats, L. Hadjarapoglou, Heterocycles, 39(1), 219, (1994), DOI: 10.3987/COM93-S(B) 16

24. B.P. Bandgar, S.S. Pandit, Journal of Sulfur Chemistry, 25(5), 343(2004), DOI: 10.1080/17415990412331317928.

25. S.B. Bandgar, B.P. Bandgar, B.L. Korbad, S.S. Sawant, Tetrahedron Letters, 48(7), 1287(2007), DOI: $10.1016 / j$.tetlet.2006.12.024

26. (a) H. Maeta, T. Nagasawa, Y. Handa, T. Takei, Y. Osamura, K. Suzuki, Tetrahedron Letters, 36(6), 899(1995), DOI:10.1016/0040-4039(94)02332-6 (b) C. K.Z. Andrade, N.R. Azevedo, Tetrahedron Letters, 42(37), 6473(2001), DOI:10.1016/S0040-4039(01)01306-5 (c) J. Howarth, K. Gillespie, Tetrahedron Letters, 37(33), 6011(1996), DOI: 10.1016/j.tetlet.2007.09.140 (d) M. Yamamoto, M. Nakazawa, K. Kishikawa, S. Kohmoto, Chemical Communications, 1996(20), 2353 (1996), DOI:10.1039/CC9960002353

27. Q. Guo, T. Miyaji, R. Hara, B. Shen, T. Takahashi, Tetrahedron, 58(36), 7327(2002), DOI: 10.1016/S0040-4020(02)00738-X

28. K. Suzuki, T. Hashimoto, H. Maeta, T. Mastumoto, Synlett, 1992(2), 125(1992), DOI: 10.1055/s-1992-21288

29. (a) S. Arai, Y. Sudo, A. Nishida, Synlett, 2004(6), 1104, (2004), DOI:10.1055/s-2004-817766 (b) A. Ortiz, L. Quintero, H. Herna'ndez, S. Maldonado, G. Mendoza, S. Berne`s, Tetrahedron 


\section{RASĀYAN J. Chem.}

Vol. 12 | No. 4 |1997 - 2004| October - December | 2019

Letters, 44(6), 1129(2003), DOI:10.1016/S0040-4039(02)02837-X (c) C.K.Z. Andrade, R.A.F. Motos, Synlett, 2003(8), 1189(2003), DOI: 10.1055/s-2003-39902

30. J.R. Desmurs, M. Labrouille`re, C.L. Roux, H. Gaspard, A. Laporterie, L.J. Dubac, Tetrahedron Letters, 38(51), 8871(1997), DOI:10.1016/S0040-4039(97)10401-4

31. (a) S. Kobayashi, S. Iwamoto, Tetrahedron Letters, 39(26), 4697(1998), DOI:10.1016/S00404039(98)00881-8 (b) M.J. Earle, U. Hakala, B.J. McAuley, M. Nieuwenhuyzen, A. Ramani, K.R. Seddon, Chemical Communications, 2004(12), 1368(2004), DOI:10.1039/B403650F (c) S. Kobayashi, K. Arai, H. Shimizu, Y.Ihori, H. Ishitani, Y. Yamashita, Angewandte Chemie Int. Ed., 44(5), 761(2005), DOI: 10.1002/anie.200462204

32. A. Kawada, S. Mitamura, M. Jun-ichi,T. Tsuchiya, S. Kobayashi, Bulletin of the Chemical Society of Japan, 73(10), 2325(2000), DOI:10.1246/bcsj.73.2325

[RJC-5381/2019] 